\title{
Schon15 Minuten Bewegung täglich verlängern das Leben
}

\section{Es gibt viele Hinweise darauf, dass regelmäßige körperliche Betätigung das Risiko vaskulärer Erkrankungen reduziert. Empfohlen werden 150 Minuten Aktivität pro Woche. Unbe- kannt ist bisher, ob bereits weniger Bewegung vaskuläre Ereignisse und andere Todesursachen reduzieren kann.}

— In einer prospektiven Kohortenstudie wurden 416175 Einwohnern von Taiwan in den Jahren von 1996 bis 2008 gescreent. Im Schnitt wurden die 199265 Männer und 216910 Frauen über einen Zeitraum von acht Jahren beobachtet. Die Betroffenen gaben mithilfe eines Fragebogens an, wie häufig pro Woche sie sich körperlich betätigten oder Sport trieben. Aus den Angaben zur Intensität und Dauer der körperlichen Aktivität wurden die Patienten in fünf verschiedene Gruppen eingeteilt. Zusätzlich wurde noch unterschieden, ob die Aktivität sehr intensiv war. Der Endpunkt war die Hazard-Ratio für Sterblichkeit und die Lebenserwartung.
Verglichen mit Personen, die sich körperlich nicht betätigen, führte bereits eine körperliche Aktivität von 92 Minuten pro Woche oder $15 \mathrm{Mi}$ nuten pro Tag zu einer 14\%igen Reduktion der Sterblichkeit und zu einer Verlängerung der Lebenserwartung von drei Jahren. Zusätzliche 15 Minuten pro Tag körperliche Betätigung reduziert die Sterblichkeit um weitere $4 \%$ und die Sterblichkeit an malignen Tumoren um 1\%. Diese Ergebnisse waren unabhängig von Altersgruppen und Geschlecht und ebenfalls unabhängig von kardiovaskulären Risikofaktoren.

Körperliche Aktivität von 15 Minuten pro Tag reduziert bereits signifikant die Sterblichkeit, verlängert die Lebenserwartung und ist insbesondere bei Menschen mit einem erhöhten Risiko für kardiovaskuläre Erkrankungen wirksam.

\section{- C. P. Wen et al.}

Minimum amount of physical activity for reduced mortality and extended life expectancy: a prospective cohort study. Lancet 378 (2011) 1244-1253

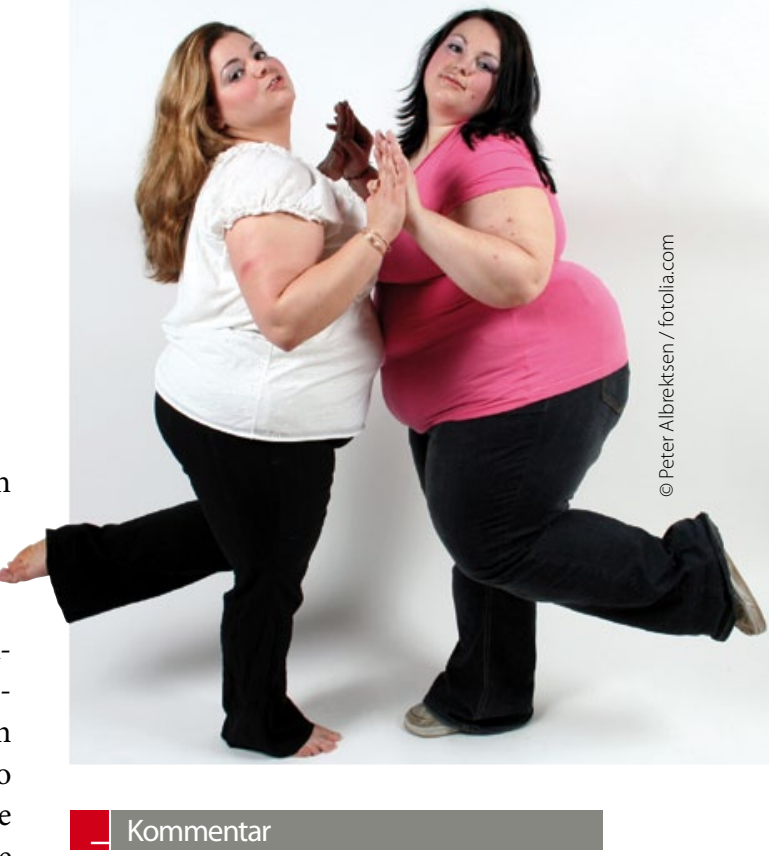

Diese Studie wurde in Taiwan durchgeführt und muss nicht notwendigerweise für Europäer gelten. Sie ist allerdings in vieler Hinsicht bemerkenswert:

1. Bereits 15 Minuten körperliche Aktivität am Tag senkt die Mortalität und verlängert die Lebenserwartung.

2. Es besteht eine fast lineare Beziehung zwischen Dauer und Intensität von körperlicher Aktivität und Reduktion der Mortalität.

3. Durch körperliche Aktivität wird nicht nur, wie zu erwarten, das Risiko von kardiovaskulären Erkrankungen reduziert, sondern überraschenderweise auch das Risiko für fast alle malignen Tumoren.

H.-C. DIENER = 